\title{
Picosecond dynamics of electronic energy transfer in condensed phases ${ }^{a)}$
}

\author{
D. P. Millar, ${ }^{\text {b) }}$ R. J. Robbins, and A. H. Zewail') \\ Arthur Amos Noyes Laboratory of Chemical Physics') California Institute of Technology, Pasadena, \\ California 91125 \\ (Received 29 April 1981; accepted 24 June 1981)
}

\begin{abstract}
Energy transfer between donor and acceptor molecules randomly distributed in condensed phases is investigated by time-resolved spectroscopy on the picosecond and nanosecond time scales. The effects of translational diffusion and excitation transfer among the donors is experimentally observed and used to test theoretical models based on a diffusion equation for the donor excitation. The time-resolved data demonstrate that the Förster dipole-dipole model is valid in the cresyl violet (donor):azulene (acceptor) system from 1 ps to at least $10 \mathrm{~ns}$ after excitation, and over a 1000-fold range of acceptor concentration. The critical transfer distance obtained from the transient experiments $(26.6 \AA)$ is in excellent agreement with the value obtained from the spectral overlap $(27.8 \AA)$ at all acceptor concentrations. In fluid solutions the donor decay agrees very well with the approximate solution of the diffusion equation including a sink term for energy transfer. The deviations observed at high donor concentrations suggest that donor-donor excitation transfer is nondiffusive on the picosecond time scale.
\end{abstract}

\section{INTRODUCTION}

The transfer of electronic excitation energy in condensed phases has been a subject of interest since the pioneering work of Förster. ${ }^{1}$ Most experimental work in this field has focused on the role of multipolar ${ }^{1,2,3}$ and exchange $e^{2,3}$ interactions in electronic excitation transfer. Time-resolved studies are particularly useful in this respect since the time dependence of the excited donor (or acceptor) population depends, in part, on the spatial dependence of the interaction between a single donor and acceptor. The first time-resolved experiments of singlet-singlet energy transfer, ${ }^{4}$ which were restricted to times longer than several nanoseconds, found general agreement with the dipole-dipole resonance transfer model, originally developed by Förster, ${ }^{1}$ and later by Dexter, ${ }^{2}$ Galanin, ${ }^{5}$ and others. ${ }^{8}$ The extension to the picosecond time domain was first achieved by Rehm and Eisenthal. ${ }^{7}$ By monitoring the anisotropy of the transient ground-state absorption of rhodamine $6 \mathrm{G}$ (donor) following excitation with a picosecond pulse, the time decay of the excited donor population in the presence of malachite green (acceptor) was measured. These authors found that the decay agreed with the Förster dipole-dipole model from the earliest times they could measure, 20 ps. Furthermore, the critical transfer distance inferred from the decay, 53 $\AA$, was in approximate agreement with that calculated from the spectral overlap, $48 \AA .^{7}$ More recently, Porter and Tredwell ${ }^{3}$ have examined the rhodamine $6 \mathrm{G}$ (Rh6G) : malachite green (MG) system using a picosecond streak camera to time resolve the donor fluorescence. They found good agreement with the Förster model and obtained $R_{0}=52.5 \AA$ in a series of mixtures containing between $1.0 \times 10^{-3} \mathrm{~mol} \ell^{-1}$ and $1.0 \times 10^{-2} \mathrm{~mol} \ell^{-1}$

a) This work was supported in part by a grant from the National Science Foundation (DMR-8105034), and from the Department of Energy.

b) Fulbright-Hays Graduate Student.

${ }^{c}$ Alfred P. Sloan Fellow and Camille and Henry Dreyfus Foundation Teacher-Scholar.

${ }^{\text {d) }}$ Contribution No. 6410 . of MG. These authors found that the Förster model was valid from as early as 10 ps after excitation, the limit of their time resolution. ${ }^{8}$

Adams et al. ${ }^{9}$ subsequently studied donor-acceptor electronic energy transfer in the DODCI (donor) : MG (acceptor) and DODCI : DQOCI systems, using a synchroscan streak camera to time resolve the donor fluorescence. While these authors found that the decay curves were of the form expected from the Förster model (see Sec. II), they also found that the apparent critical transfer distance increased rapidly with decreasing acceptor concentration, which these authors took to imply a breakdown of the Förster model at low acceptor concentrations. At the higher acceptor concentrations the $R_{0}$ values attained constant values, comparable in magnitude with the values measured by Rehm and Eisenthal, ${ }^{7}$ and Porter and Tredwell ${ }^{8}$ for Rh6G : MG under similar conditions. The apparent breakdown of the Förster model at large donor-acceptor separations is puzzling, since it is in this regime that the model is expected to be valid. ${ }^{6}$

On the theoretical side, attention has recently been directed towards the effect of diffusion on donor-acceptor energy transfer. ${ }^{10-15}$ Because of the strong distance dependence of multipolar transfer rates, the mobility of $\mathrm{D}^{*}$ and $\mathrm{A}$ (donor and acceptor) due to translational diffusion causes a faster decay of the excited donors than expected from the simple Förster model. The generalization of the Förster model to include diffusion was first considered by Yokota and Tanimoto, ${ }^{18}$ and more recently in a simpler approach by Gösele et al. ${ }^{13-15}$ These authors found approximate analytical solutions to the diffusion equation describing the donor excitation with a sink term for the D-A energy transfer. More recently Butler and Pilling ${ }^{10}$ solved this equation numerically; the calculations showed the expected deviations from simple Förster kinetics and were in excellent agreement with the approximate analytic solution derived by Gösele $e t$ al. ${ }^{13-15}$ However, these equations have not yet been experimentally tested on the picosecond timescale. 
Donor-donor excitation energy transfer also results in mobility of the excited donors and causes a faster quenching by the acceptors. Recent theoretical interest in the problem of electronic excitation transfer between identical molecules in disordered systems ${ }^{11,17}$ has focused on whether or not the migration can be described by a diffusion equation. Förster first showed that electronic excitation transfer on an ordered cubic array, by the dipole-dipole mechanism, is diffusive at all times. ${ }^{18}$ Recently Haan and $\mathrm{Zwanzig}^{19}$ considered the effect of disorder; they found that the transfer is nondiffusive at early times in dilute solutions and conjectured that diffusive behavior is approached only at long times or at high concentrations. Gochanour et al. ${ }^{20}$ subsequently expanded on this treatment by including high order terms in the diagrammatic expansion of the Green function. The time-dependent diffusion coefficients calculated by Gochanour et al. ${ }^{20}$ showed the nondiffusive behavior at low concentrations and the diffusive behavior at long times (see Fig. 10 of Ref. 20). The quenching of excited donors by acceptors can serve as a probe for this excitation transfer among the donors, and so test whether it is diffusive or not on the time scale of the D-A energy transfer.

It is the purpose of this paper to experimentally investigate these questions by time-resolved spectroscopy on the picosecond and nanosecond timescales. We test the Förster model in the cresyl violet: azulene system in both fluid and viscous solvents. Our approach is to measure the time decay of the excited donors using both ground state recovery (GSR) and time-correlated single photon counting techniques: GSR probes the early time dynamics with approximately 1 ps resolution while photon counting allows the donor fluorescence decay to be measured over a wide range of acceptor concentrations. These time-resolved data show that the Förster model is valid from 1 ps to $10 \mathrm{~ns}$ after excitation and over a 1000-fold range of acceptor concentration. The critical transfer distance is constant over the entire concentration range, in contrast with the results of Adams et al. ${ }^{9}$ The effect of donor-donor excitation transfer is experimentally observed and strongly suggests that donor transport is nondiffusive on the picosecond timescale.

\section{THEORY}

In this section we will briefly outline the Förster model and present equations for the time-dependent excited donor population. Then the additional effects of translational diffusion of the solutes, and excitation transfer among the donors are considered. Finally, the relationship to the observables in time-resolved experiments, and the formal equivalence of the GSR induced transmis sion signal and the time-dependent fluorescence intensity are discussed.

\section{A. Förster model}

Förster ${ }^{1}$ considered the weak coupling of the electronic and vibronic states of $D$ and $A$ by a dipole-dipole interaction, and showed that the rate constant for energy transfer between a single donor and acceptor separated by a distance $R$ could be written as

$$
K_{\mathrm{DA}}=\frac{3}{2} K^{2}\left(1 / \tau_{\mathrm{D}}\right)\left(R_{0} / R\right)^{6},
$$

where $\kappa$ contains the angular dependence of the dipoledipole interaction;

$$
\kappa^{2}=\left(\sin \theta_{D} \sin \theta_{A} \cos \phi-2 \cos \theta_{D} \cos \theta_{A}\right)^{2},
$$

where $\theta_{D}$ and $\theta_{A}$ are the angles specifying the direction of the transition moments $\left(S_{1}-S_{0}\right)$ of $\mathrm{D}$ and $\mathrm{A}$ with respect to the vector joining $\mathrm{D}$ and $\mathrm{A} ; \phi$ is the azimuthal angle between $D$ and $A ; \tau_{D}$ is the excited donor lifetime in the absence of acceptors, and $R_{0}$ is the critical transfer distance. It is related to the spectral overlap of $D$ and $\mathrm{A}$ by the equation

$$
R_{0}^{6}=\frac{9000 \ln 10 \Phi_{\mathrm{D}}\left(\frac{2}{3}\right)}{128 \pi^{5} n^{4} N} \int_{0}^{\infty} \frac{F_{\mathrm{D}}(\bar{\nu}) \epsilon_{\mathrm{A}}(\bar{\nu})}{\bar{\nu}^{4}} d \bar{\nu},
$$

where $N$ is Avogardro's number, $\Phi_{\mathrm{D}}$ is the quantum yield of donor emission, $n$ is the solvent refractive in$\operatorname{dex}, \epsilon_{\mathrm{A}}(\bar{\nu})$ is the acceptor molar decadic extinction coefficient at wavenumber $\bar{\nu}$, and $F_{\mathrm{D}}(\bar{\nu})$ is the donor fluorescence intensity at $\bar{\nu}$ (normalized to unit area on a wavenumber scale). $R_{0}$ can thus be calculated from the known spectral properties of $\mathrm{D}$ and $\mathrm{A}$.

Consider the differential equation governing the decay of an individual donor molecule $i$ in an ensemble of $N_{\mathrm{A}}$ acceptors

$$
\frac{d \rho_{i}}{d t}=-\rho_{i} / \tau_{\mathrm{D}}-1 / \tau_{\mathrm{D}} \sum_{j=1}^{N_{\mathrm{A}}} \frac{3}{2} \kappa_{i j}^{2}\left(R_{0} / R_{i j}\right)^{6} \rho_{i},
$$

where $\rho_{i}(t)$ is the probability that the donor molecule $i$ is excited at time $t$. The sum extends over all acceptors $j$, and depends on the relative separations $R_{i j}$ and orientations $\kappa_{i j}$. Back transfer from the excited acceptors to the donors is ignored in Eq. (4), which is valid when the donor and acceptor are different species. It follows that the individual donor decay is exponential. However, it is not the decay of a single donor that is observed in a time-resolved experiment, but rather the decay of an ensemble of donors with different distributions of $R_{i j}$ and $\kappa_{i j}$. Several authors have considered the procedure for evaluating the ensemble averaged decay law. 1,5,6,21-24 Gösele et al. ${ }^{21}$ showed that Förster's statistical averaging method, ${ }^{1}$ which has been criticized by some authors, ${ }^{22,23}$ is a correct procedure and that it is in agreement with other methods based on averaging of the differential equation ${ }^{5}$ or on pair probabilities. ${ }^{21,25}$ Förster averaged each of the $N_{\mathrm{A}}$ exponential decay terms in the solution of Eq. (4). For a random distribution of $R_{i j}$ and $\kappa_{i j}$, the average is independent of which acceptor is considered and the ensemble averaged donor decay probability is a product of $N_{\mathrm{A}}$ identical terms

$$
\begin{aligned}
\rho(t)= & e^{-t / \tau_{\mathrm{D}}}\left[\iiint \int \exp \left(-\frac{3}{2} \kappa^{2}\left(R_{0} / R\right)^{6} t / \tau_{\mathrm{D}}\right)\right. \\
& \left.\times \frac{R^{2} \sin \theta_{\mathrm{D}} \sin \theta_{\mathrm{A}}}{2 V} d \theta_{\mathrm{D}} d \theta_{\mathrm{A}} d \phi d R\right]^{N_{\mathrm{A}}},
\end{aligned}
$$

where $V$ is the volume of the ensemble. The final result is ${ }^{1,5,6,21}$

$$
\rho(t)=\exp \left[-t / \tau_{\mathrm{D}}-g \frac{4}{3} \pi^{3 / 2} n_{\mathrm{A}} R_{0}^{3}\left(t / \tau_{\mathrm{D}}\right)^{1 / 2}\right],
$$

where $R_{0}$ is the critical transfer distance defined in Eq. (3), and $n_{A}$ is the acceptor number density, $g$ is a nu- 
merical factor given by

$$
g=\left(\frac{3}{2}\left\langle\kappa^{2}\right\rangle\right)^{1 / 2} \text {, }
$$

where $\left\langle\kappa^{2}\right\rangle$ is an averaged orientation factor, and depends on how the orientations are averaged. In the "dynamic averaging limit" 26 molecular reorientation is infinitely rapid, all possible orientations are sampled during the energy transfer, and the orientation factors [Eq. (2)] can be preaveraged; the result is $\kappa_{i j}^{2}=2 / 3$ for all $i$, $j(g=1)$, which is then used in Eq. (1) and the subsequent ensemble average. In the "static averaging limit" 26 the orientations are fixed throughout the energy transfer, and the orientation terms must be ensemble averaged over a random (but static) angular distribution as done in Eq. (5); then $g=0.845 .{ }^{27,28}$ In the intermediate regime, $g$ will be a function of the rotational diffusion coefficients of $D$ and $A$ and the acceptor concentration. Bojarski and Dudkiewicz ${ }^{29}$ recently derived an approximate equation for $\left\langle\kappa^{2}\right\rangle$ based on an interpolation between the two limits described above. Note that the effect of rotational mobility is quite minor, resulting in at most a $15 \%$ reduction of the quenching term in $\mathrm{Eq}$. (6).

\section{B. Effect of translation diffusion}

The derivation of the donor decay law [Eq. (6)] as sumes that the distribution of $R_{i j}$ is time-independent. This will not be true in fluid solutions, and deviations from the decay law are expected. ${ }^{10}$ Several authors have considered this problem theoretically by adding a diffusion term to the differential equation for the donor excitation probability [Eq. (4)]. ${ }^{10-16}$ The resulting equation was first solved by Yokota and Tanimoto ${ }^{16}$ using a method of Padé approximants. Their result for $\rho(t)$ is complex, and not the most suitable form for curve-fitting. More recently, Gösele et al. ${ }^{13-15}$ obtained an approximate solution, based on an interpolation between the known solutions at early and long times. They showed that when long range resonance transfer dominates, the donor decay law is ${ }^{13,14}$

$\rho(t)=\exp \left[-t / \tau_{\mathrm{D}}-4 \pi D r_{F} n_{\mathrm{A}} t-g \frac{4}{3} \pi^{3 / 2} n_{\mathrm{A}} R_{0}^{3}\left(t / \tau_{\mathrm{D}}\right)^{1 / 2}\right]$,

where $r_{F} \approx 0.676\left(g^{2} R_{0}^{6} / \tau_{D} D\right)^{1 / 4}$ is an "effective trapping radius" and $D$ is the relative diffusion coefficient $\left(D=D_{A}\right.$ $\left.+D_{\mathrm{D}}\right)$. This result is valid whenever $z_{0}=\left(1 / 2 r_{\mathrm{AD}}^{2}\right)$. $\left(g^{2} R_{0}^{6} / \tau_{\mathrm{D}} D\right)^{1 / 2} \gg 1$, where $r_{\mathrm{AD}}$ is the hard-sphere encounter distance. Later it will be shown that this condition is satisfied in our experiments. Gösele et al. ${ }^{13}$ state that this relatively simple equation is as accurate as the more complex solution obtained by Yokota and Tanimoto. ${ }^{18}$

The only additional parameter appearing in $\mathrm{Eq}$. (8) is the diffusion coefficient. For translational diffusion, $D$ may be estimated from classical hydrodynamics, using the Stokes-Einstein equation ${ }^{30}$

$$
D_{T}=\frac{k T f_{\mathrm{A}}}{6 \pi \eta r_{\mathrm{A}}}+\frac{k T f_{\mathrm{D}}}{6 \pi \eta r_{\mathrm{D}}},
$$

where $r_{\mathrm{A}}$ and $r_{\mathrm{D}}$ are the radii of the equivalent spheres of the donor and acceptor, respectively, and $f_{\mathrm{A}}$ and $f_{\mathrm{D}}$ are shape factors. The subscript $T$ denotes a translational diffusion coefficient.

\section{Effect of donor-donor excitation transfer}

When the absorption and fluorescence of the donor overlap, long-range resonance transfer between excited and unexcited donor molecules will occur. In most cases the $D-D$ rate will be of the same form as the $D-A$ rate [Eq. (1)] with a critical transfer distance $\mathbf{R}_{0}$ dependent on the self-overlap. When the donor concentration is sufficiently dilute that the average donor separation is much larger than $R_{0}$, then the treatment of $D-A$ energy transfer given above is correct. However, when the $\mathrm{D}-\mathrm{D}$ and $\mathrm{D}-\mathrm{A}$ rates are comparable, the situation is more complicated. The problem of simultaneous D-D and $D-A$ energy transfer has long been of interest in exciton trapping in solids. ${ }^{31}$ Here two limits are considered, depending on the relative rates of $D-D$ and $D-A$ transfer. When D-A transfer dominates, the $\mathrm{D}-\mathrm{D}$ transfer is included by adding diffusion terms to the donor rate equations, as done in the case of translational diffusion. Then Eq. (8) would be appropriate with $D$ determined by exciton diffusion. This is a sensible procedure in an ordered solid, for which the D-D transfer (by a dipole-dipole mechanism) is diffusive at all times. ${ }^{18}$ (That is, the mean square displacement of the excitation during the excited state lifetime is linear in time and the diffusion coefficient is time-independent.) However, in a dilute solution, the effect of the disorder is to make the donor excitation transfer nondiffusive over most times of experimental interest. ${ }^{10,20}$ In this case the diffusion coefficient is time-dependent and Eq. (8) would not apply since it assumes a constant diffusion coefficient.

The results of Gochanour et al. ${ }^{20}$ imply that diffusive behavior is attained at long times, with a limiting diffusion coefficient given by

$$
D_{E}=0.428 C^{4 / 3} \mathrm{R}_{0}^{2} \tau_{D}^{-1}
$$

where $C$ is a reduced concentration defined by

$$
C=4 \pi n_{\mathrm{D}} \mathbf{R}_{0}^{3} / 3
$$

and $n_{\mathrm{D}}$ is the number density of donors. The subscript $E$ denotes an excitation diffusion coefficient. Haan and Zwanzig $^{19}$ found a similar equation for the limiting diffusion coefficient, but with a numerical factor of 0.324 . Equation (10) is considered to be more accurate, since Gochanour et al. included higher order terms in the expansion of the Green's function. ${ }^{20}$

If diffusive donor excitation transfer is achieved on the time scale of the D-A transfer, then Eq. (8) will apply with $D$ given by Eq. (10). Nondiffusive transport should result in deviations from this decay law.

\section{Experimental observables}

In the ground state recovery experiment a fraction of the donor ground state population is excited to the $S_{1}$ state, causing a decrease in the ground-state absorption, which will decay to zero as the excited donors subsequently relax by D-A energy transfer and intramolecular processes. The relevant observable is the change in the ground state transmission at the probe wavelength. 


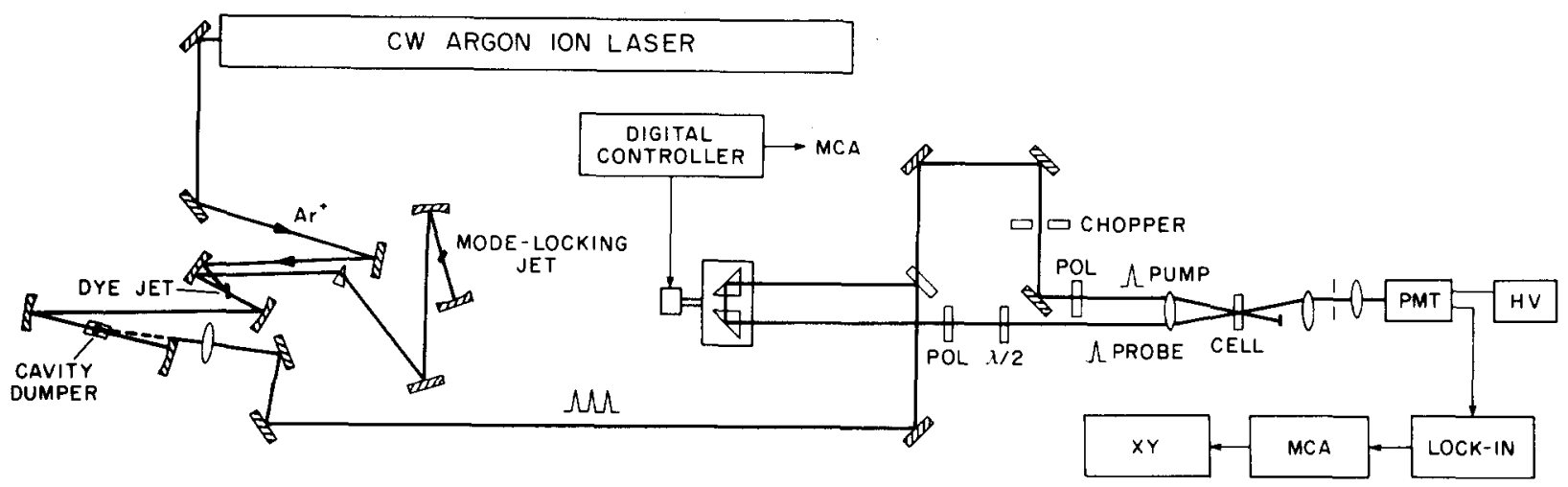

FIG. 1. Experimental setup for ground state recovery (GSR) measurements. The passively mode-locked Rh6G dye laser is shown to the left of the figure. The interferometer and signal processing electronics are shown in the right half of the figure. See text for details. $\mathrm{POL}=$ polarizer,$\lambda / 2=$ half-wave plate, $\mathrm{PMT}=$ photomultiplier, $\mathrm{HV}=$ high voltage power supply, LOCK-IN $=1$ lock-in amplifier, $\mathrm{MCA}=$ multichannel analyzer, $\mathrm{XY}=\mathrm{XY}$ recorder. as

The ground state absorption coefficient may be written

$$
\alpha(t)=a \iint n_{\mathrm{D}}(\theta, \phi, t) f^{2}(\theta, \phi) \sin \theta d \theta d \phi,
$$

where $n_{\mathrm{D}}(\theta, \phi, t)$ is the density of ground state donor molecules with their transition dipoles oriented $(\theta, \phi)$ with respect to a laboratory fixed coordinate system, $f(\theta, \phi)$ is the projection of the dipole onto the polarization direction of the probe light, and $a$ is a constant. Considering only a single ground and excited state (twolevel approximation), and assuming that molecular reorientation is the same in the two states, we can write

$$
n_{\mathrm{D}}(\theta, \phi, t)=\frac{n_{\mathrm{D}}}{4 \pi}-n_{\mathrm{D}} *(\theta, \phi, t),
$$

where $n_{D^{*}}(\theta, \phi, t)$ is the density of excited donors. The initial photo-selected distribution of the excited dipoles evolves in time due to reorientation and also decays due to energy transfer and intramolecular processes. If these processes are independent, then

$$
n_{\mathrm{D}} *(\theta, \phi, t)=n_{\mathrm{D}} *(0) W(\theta, \phi, t) \rho(t),
$$

where $\rho(t)$ is the mean donor decay probability, defined in Sec. II A, $n_{D *}(0)$ is the total density of excited donors at zero time (excitation) and $W(\theta, \phi, t)$ is the normalized angular distribution of the excited dipoles. ${ }^{32}$

What is measured in the GSR experiment is the change in transmission, given by

$$
\begin{aligned}
\Delta T(t) & =T(t)-T_{0} \\
& =e^{-\alpha_{0} \ell}\left(e^{-\Delta \alpha(t) \ell}-1\right) \\
& \simeq-T_{0} \ell \Delta \alpha(t),
\end{aligned}
$$

where $T_{0}$ is the small-signal transmission. The approximation in the last step is valid for small transmission changes, which is true in the present work, and assures that the GSR signal is linear in $\Delta \alpha(t)$.

The small signal absorption coefficient $\alpha_{0}$ is identified as the angular integral of the first term on the right hand side of Eq. (13). The change in absorption $\Delta \alpha(t)$ involves the integral of the second term in Eq. (13). Combining Eqs. (11) through (15), we arrive at the final expression for the GSR signal

$$
\Delta T(t)=b \iint W(\theta, \phi, t) f^{2}(\theta, \phi) \sin \theta d \theta d \phi \rho(t),
$$

where $b$ is a constant.

In the fluorescence experiment, the instantaneous fluorescence intensity is measured at time $t$ after excitation through an analyzing polarizer. It has been shown ${ }^{32,45}$ that the fluorescence intensity $I(t)$ can be written in the same form as $\mathrm{Eq}$. (16), where $f$ now refers to the projection of the dipole $(\theta, \phi)$ onto the analyzing direction (and the constant $b$ is different).

The GSR and fluorescence experiments are thus formally equivalent when the assumptions leading to Eqs. (13) and (14) are valid. Both $\Delta T(t)$ and $I(t)$ are linearly proportional to the donor excitation probability $\rho(t)$. Either signal may be used to measure $\rho(t)$.

Finally, it is important to note that Eq. (16) contains an additional time dependence due to molecular rotation. As is well known in the fluorescence depolarization problem, ${ }^{33}$ the effect of $W(\theta, \phi, t)$ can be suppressed by using an appropriate linear polarization direction for the analyzer (or probe light in GSR): when this direction is $54.7^{\circ}$ from the polarization direction of the excitation pulse, $\Delta T(t)$ and $I(t)$ become independent of $W(\theta, \phi, t) .^{33-35}$

\section{EXPERIMENTAL}

\section{A. Ground state recovery}

The setup used for ground state recovery experiments is shown in Fig. 1. Picosecond pulses were generated by a passively mode-locked rhodamine $6 \mathrm{G} \mathrm{cw}$ dye laser, shown to the left in Fig. 1. The laser is similar in design to that described by Ippen and Shank, ${ }^{36}$ consisting of a triple-folded seven-mirror optical resonator, an intracavity saturable absorber jet stream, and an acousto-optic cavity dumper. The dye laser was pumped by the $5145 \AA$ line of a cw argon ion laser. The cavity dumped output consisted of a continuous train of pulses with selectable repetition frequency, typically $100 \mathrm{kHz}$. The pulse duration, measured by second harmonic generation autocorrelation techniques, ${ }^{37}$ was typi- 
cally between 0.5 and 2.0 ps. $^{34}$ The pulse energy was $3 \mathrm{~nJ}$, corresponding to a peak power of a few kilowatts.

The vertically polarized dye laser output was divided in a $95: 5$ ratio in a Michelson interferometer, and a variable delay was introduced by the prism pair mounted on a stepper motor driven translation stage. The parallel and noncollinear output beams from the interferometer were focused to a common spot in a thin cell of the donor-acceptor mixture. The weaker, delayed beam (probe) passed through an aperture to a photomultiplier. A half-wave plate was used to rotate the probe polarization $54.7^{\circ}$ from vertical. The strong beam was modulated by a mechanical chopper before the sample, and was blocked from passing to the PMT. The modulation of the probe beam intensity at the chopping frequency was phase-sensitively detected by a lock-in amplifier. This signal was proportional to the transmission change resulting from excitation by the strong beam. The lockin output was digitized by a voltage-to-frequency converter and stored in the memory of a multichannel analyzer as a function of path delay introduced by the stepper motor. The signals were averaged by repetitive scanning of the desired delay interval.

\section{B. Fluorescence decay experiments}

Fluorescence decays were measured by the time-correlated single photon counting technique. ${ }^{38}$ Samples were excited with the vertically polarized pulse train from a synchronously pumped mode-locked Rh6G dye laser (duration $\sim 3 \mathrm{ps}$ ). The dye laser output was modulated by an acousto-optic modulator to reduce the repetition frequency of the excitation beam to $1 \mathrm{MHz}$. The fluorescence was collected at right angles and passed through an analyzing polarizer set $54.7^{\circ}$ from vertical. Cutoff filters isolated the donor fluorescence from scattered laser light. Fluorescence from the acceptor, azulene, has very low quantum efficiency ${ }^{39}$ and did not interfere with the donor fluorescence. A photodiode sampled a small fraction of the excitation beam and triggered a constant fraction discriminator (ORTEC 473A), whose output provided a "start" pulse for a timeto-amplitude converter (TAC, ORTEC 457). Single photon pulses from the photomultiplier (Amperex XP2020Q) triggered a second constant fraction discriminator (ORTEC 583) which stopped the TAC. The TAC output voltages, proportional to the interval between start and stop pulses, were stored in a pulse height analyzer (Tracor Northern TN1706). Counting rates below 1\% of the excitation rate were used. A brief description of this apparatus has appeared ${ }^{40}$ and a complete description will appear in a forthcoming publication. ${ }^{41}$

The instrument response width was $250 \mathrm{ps,} \mathrm{mainly}$ due to dispersion in photoelectron transit times in the photomultiplier, resulting in a distortion of the decay curves near zero time due to convolution with the response function. However, this time range was readily probed by GSR with $\sim 1$ ps time resolution. Therefore most of the fluorescence decay curves were analyzed beginning $\approx 500$ ps after excitation where convolution was negligible. Identical results were obtained when convolution was included in the fitting procedure. De-

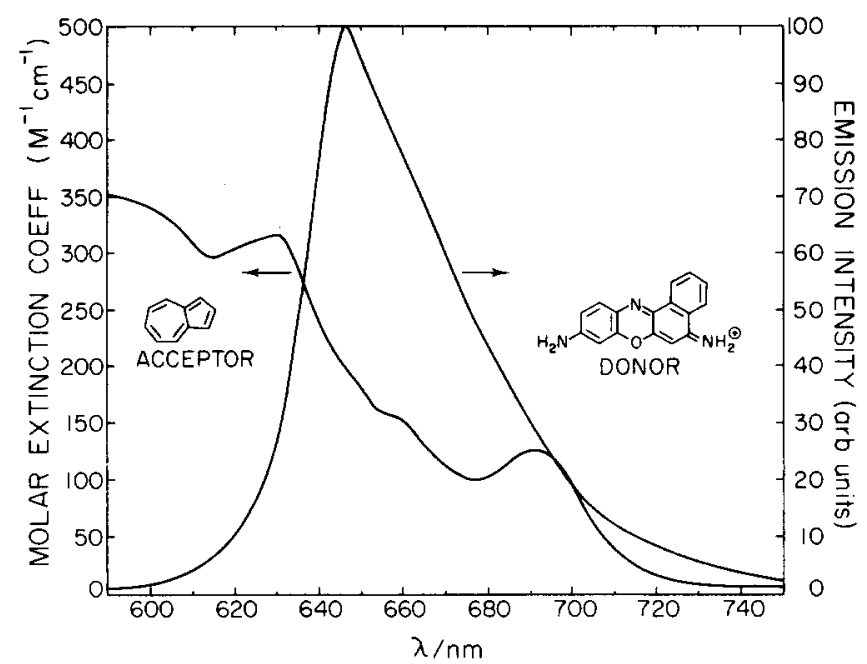

FIG. 2. Steady-state spectra and chemical structures of cresyl violet (donor) and azulene (acceptor). The donor fluorescence spectrum and acceptor absorption spectrum are shown in the region of overlap.

convolution was only required at the highest acceptor concentration $\left(4 \times 10^{-2} \mathrm{~mol} \ell^{-1}\right)$.

\section{Steady state spectra}

Absorption spectra of cresyl violet and azulene were recorded on a Cary 14 spectrophotometer. The emission spectrum of cresyl violet was recorded on a Perkin Elmer MPF-3 spectrofluorimeter. Spectra were digitized and input to a computer for numerical integration.

\section{Materials}

Cresyl violet perchlorate (laser grade) and azulene (99\%) were obtained from Eastman and Aldrich, respectively, and used as supplied. Solutions were made up in methanol or ethylene glycol solvents at room temperature $\left(23^{\circ} \mathrm{C}\right)$. The solvent viscosities at this temperature were $0.57 \mathrm{cP}$ (methanol) and $19.5 \mathrm{cP}$ (ethylene glycol). Donor and acceptor concentrations were carefully controlled and checked by spectrophotometry.

\section{RESULTS}

\section{A. Steady state spectra}

The donor fluorescence spectrum and acceptor absorption spectrum are presented in $\mathrm{Fig.} \mathrm{2,} \mathrm{together}$ with their chemical structures. A considerable overlap of the spectra is evident. The spectral overlap integral in Eq. (3) was evaluated by numerical integration of the spectra in Fig. 2. The result was $4.5 \times 10^{-15}$ $\mathrm{cm}^{6} \mathrm{~mol}^{-1}$ in methanol and $4.3 \times 10^{-15} \mathrm{~cm}^{6} \mathrm{~mol}^{-1}$ in ethylene glycol. Using the known fluorescence quantum yield for cresyl violet in methanol, ${ }^{42} \Phi_{D}=0.545$, and other known constants, we calculated $R_{0}=27.8 \pm 0.9 \AA$ in methanol and $R_{0}=26.3 \pm 0.9 \AA$ in ethylene glycol. The cresyl violet absorption (not shown) at the excitation wavelength $(6120 \AA)$ was 200 times stronger than the azulene absorption. From the overlap of the cresyl violet absorption and fluorescence spectra, the critical 

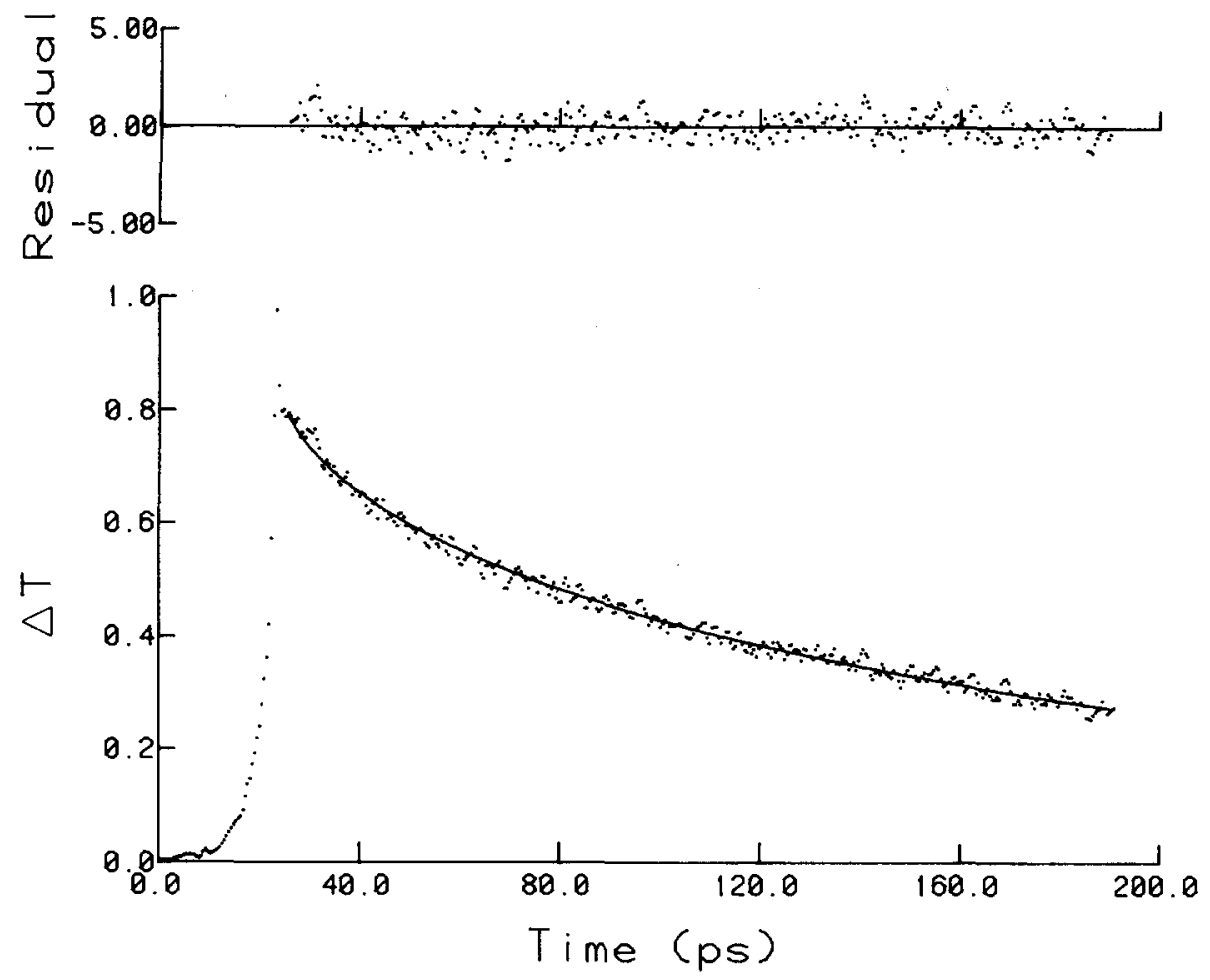

FIG. 3. Induced transmission $\Delta T$ versus probe pulse delay for cresyl violet $\left(2.0 \times 10^{-3} \mathrm{~mol}^{-1}\right)$ in methanol containing $5.0 \times 10^{-2} \mathrm{~mol} \ell^{-1}$ of azulene. The direction of polarization of the probe beam was $54.7^{\circ}$ from that of the pump beam. The vertical scale is in arbitrary units; the peak transmission change was about $3 \%$. The points are experimental data and the smooth line is the best fit of the data to Eq. (8). Shown above are the weighted residuals between the data and the best-fit curve.

transfer distance for donor-donor excitation transfer was calculated to be $R_{0}=44.5 \pm 1.5 \AA$.

\section{B. Ground state recovery}

The induced transmission change versus probe pulse delay is shown in Fig. 3 for a $2 \times 10^{-3}$ mol $\ell^{-1}$ solution of cresyl violet in methanol, containing $5.0 \times 10^{-2} \mathrm{~mol} \ell^{-1}$ of azulene. The probe polarization was $54.7^{\circ}$ from the pump direction, as discussed in Sec. IID. The buildup seen to the left of the peak in Fig. 3 is due to the finite duration of the pump and probe pulses $(1.5 \mathrm{ps}$ in this case). The sharp peak on the curves, which marks the point of coincidence of pump and probe pulses, results from the interference of the two beams. This coherent coupling artifact is present in any GSR experiment utilizing pump and probe pulses from a common laser. ${ }^{43}$

The GSR signal observed with no azulene present (not shown) was almost constant over the time range shown in Fig. 3. Longer scans revealed an exponential recovery, with $\tau_{D}=3.2 \pm 0.4 \mathrm{~ns}$ in methanol. This agreed well with the fluorescence lifetime, $3160 \mathrm{ps}$ (see later). The rate of decay of the induced transmission increased monotonically as increasing concentrations of azulene were added. The GSR curves, such as Fig. 3, were clearly nonexponential.

In Fig. 4 the GSR of a sample in ethylene glycol solvent with a considerably higher donor concentration is shown. The donor concentration was $5.0 \times 10^{-3} \mathrm{~mol} \mathrm{l}^{-1}$ and the acceptor, $4.0 \times 10^{-2} \mathrm{~mol} \ell^{-1}$.

The smooth lines in Figs. 3 and 4 are theoretical fits to Eq. (8). Translational diffusion coefficients calculated from Eq. (9) with $r_{A}=3.12 \AA$ and $r_{D}=3.72 \AA$, and corrected for nonspherical shape, were $D_{T}=2.0 \times 10^{-5}$ $\mathrm{cm}^{2} \mathrm{~s}^{-1}$ in methanol and $D_{T}=6.0 \times 10^{-7} \mathrm{~cm}^{-2} \mathrm{~s}^{-1}$ in ethylene glycol at room temperature. Excitation transfer diffusion coefficients $D_{E}$ were calculated from Eq. (10). Rotational mobility factors were calculated for each donor and acceptor concentration, using the known rotational relaxation times of cresyl violet ${ }^{34}$ in each solvent and estimating these for azulene. The donor lifetime $\tau_{D}$ was fixed in all the fits at the value measured independently in the fluorescence experiments. Equation (8) was fit to the GSR data by adjusting $R_{0}$, using a nonlinear least-squares curve fitting routine. ${ }^{44}$ The use of Eq. (8) was justified, since $z_{0}=14$ for methanol and $z_{0}=85$ for ethylene glycol (taking $r_{\mathrm{AD}}=5 \AA$ ). The reduced chisquared value, weighted by the measured variances of the data, was used to ascertain the quality of the fits. The fits were excellent in both methanol and ethylene glycol except at the highest donor concentrations.

The results of fitting the GSR data in both methanol and ethylene glycol solvents are presented in Table I. The values of $D_{T}$ and $D_{E}$ used in fitting the data for each solution are also included.

\section{Fluorescence decay data}

The isotropic $\left(54.7^{\circ}\right)$ fluores cence decays of cresyl violet were exponential in both solvents with lifetimes of $3160 \pm 15$ ps (methanol) and $2870 \pm 15$ ps (ethylene glycol). The error ranges quoted here are $95 \%$ confidence intervals. The reduced chi-squared values of the singleexponential fits were 1.0 or less. The cresyl violet concentration was $2.5 \times 10^{-7} \mathrm{~mol}^{-1}$ in all fluorescence experiments; this was necessary to prevent distortion of the decay curves due to self-absorption, which caused observable lifetime lengthening at concentrations above $10^{-8}$ mol $\ell^{-1}$. This donor concentration was almost three orders of magnitude lower than used in previous picosecond experiments, ${ }^{7-9}$ and reflects the high sensitivity 

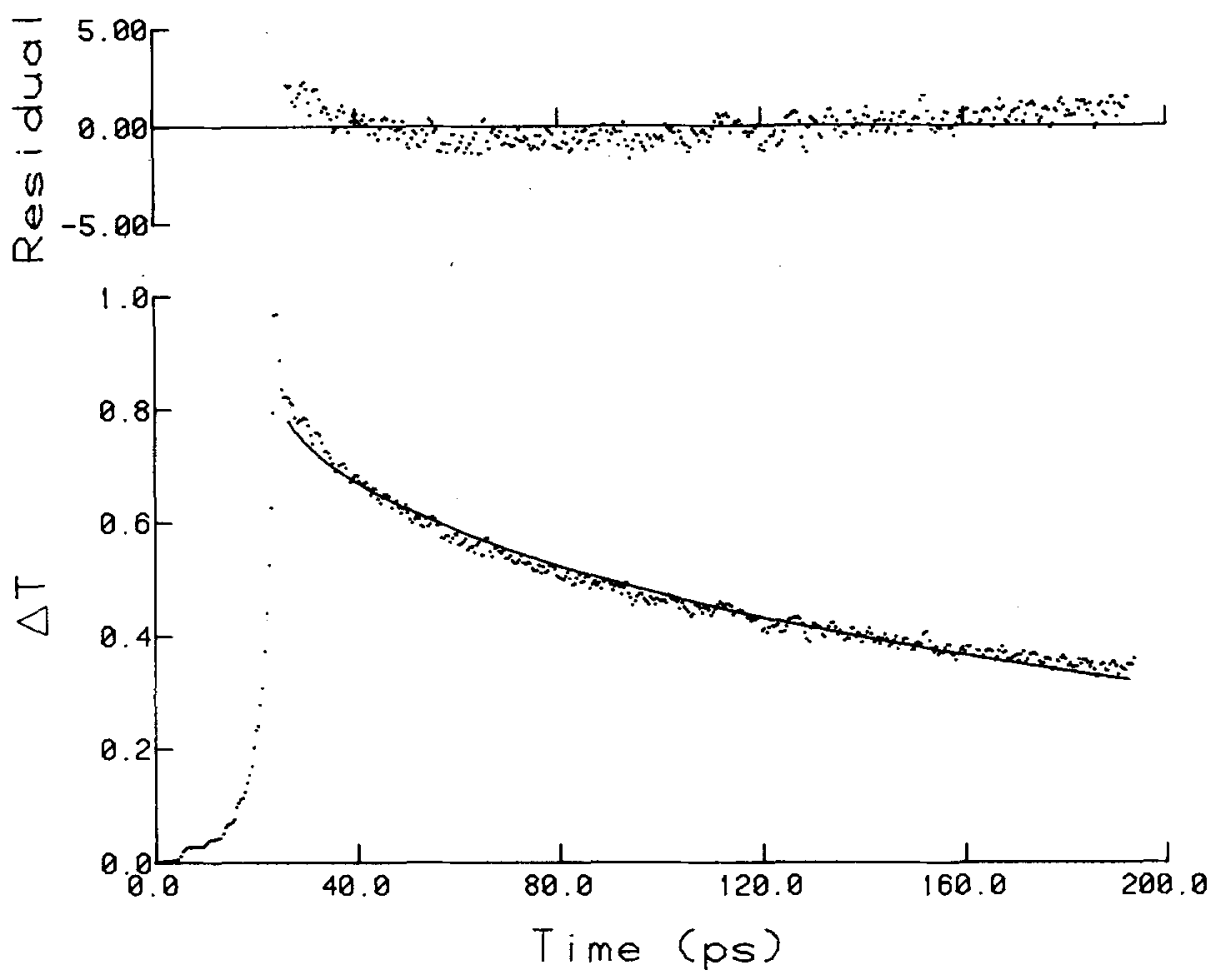

FIG. 4. Induced transmission $\Delta T$ versus probe pulse delay for cresyl violet $\left(5.0 \times 10^{-3} \mathrm{~mol} \ell^{-1}\right)$ in ethylene glycol containing $4.0 \times 10^{-2} \mathrm{~mol} \mathrm{I}^{-1}$ of azulene. The probe polarization direction was $54.7^{\circ}$ from that of the pump. The smooth line is the best fit of the data to Eq. (8). The weighted residuals are shown above. Note the deviation, which is discussed in the text.

of the single photon counting technique. The high signalto-noise ratio at this extremely low donor concentration is also apparent from the data in Figs. 5 and 6 . Donordonor excitation transfer was absent at this low donor concentration, as was verified using Eq. (10).

The fluorescence decays of cresyl violet in ethylene glycol solutions containing different concentrations of azulene are presented in Fig. 5. The fluorescence decays of cresyl violet solutions containing $1 \times 10^{-2} \mathrm{~mol} \mathrm{l^{-1 }}$ of azulene in both methanol and ethylene glycol solvents are presented in Fig. 6 . The decay curves were fit very well by $\mathrm{Eq}$. (8) over the whole range of acceptor concentrations studied, $5 \times 10^{-5} \mathrm{~mol} \ell^{-1}$ to $4 \times 10^{-2} \mathrm{~mol} \ell^{-1}$. In the fits $D_{T}$ and $\tau_{D}$ were fixed to the same values mentioned in the previous section, and $R_{0}$ was optimized us ing the same nonlinear least-squares method. The reduced chi-squared values were in the range 1.0-1.2 for all the fits, and showed no systematic trend with acceptor concentration. The results of the curve fitting are presented in Table II, where we give the $R_{0}$ values obtained at each acceptor concentration. Also included are the " $1 / e$-times," which is the time required for the fluorescence to decay to $1 / e$ of its initial intensity. These values indicate the extent of excited donor quenching by the azulene acceptors. Rotational mobility correction factors calculated for each acceptor concentration are also included in Table II.

\section{DISCUSSION}

The fluorescence decay curves of the excited donors, shown as a function of acceptor concentration in Fig. 5, display the qualitative features expected for D-A energy transfer: the curves decay faster and become more nonexponential as the acceptor concentration increases, and also decay faster in methanol than in ethylene glycol (when energy transfer dominates the decay). For the ethylene glycol solvent the decay curves were equally well fit by the no-diffusion $\mathrm{Eq}$. (6) or by Eq. (8) with $D_{T}=6.0 \times 10^{-7} \mathrm{~cm}^{2} \mathrm{~s}^{-1}$. However, the $R_{0}$ values were consistently larger by $1 \AA$ when Eq. (6) was used, indicating that translational diffusion made a small contribution to the observed energy transfer in this solvent.

TABLE I. Analysis of GSR curves.

\begin{tabular}{|c|c|c|c|c|c|c|}
\hline Solvent & $\begin{array}{l}C_{\mathrm{D}} / 10^{-3} \\
\mathrm{~mol} \mathrm{\ell ^{-1 }}\end{array}$ & $\begin{array}{l}C_{\mathrm{A}} / 10^{-2} \\
\mathrm{~mol} \tilde{T}^{-1}\end{array}$ & $\begin{array}{l}D_{T} / 10^{-5} \\
\mathrm{~cm}^{2} \mathrm{~s}^{-1}\end{array}$ & $\begin{array}{l}D_{E} / 10^{-52} \\
\mathrm{~cm}^{2} \mathrm{~s}^{-1}\end{array}$ & $R_{0} / \AA$ & $\begin{array}{l}D_{E}=0 \\
R_{0} / \check{\mathrm{A}}^{\mathrm{b}}\end{array}$ \\
\hline \multirow[t]{3}{*}{$\mathrm{MeOH}$} & 1.00 & 4.00 & 2.00 & 0.35 & $25.3 \pm 0.9$ & $25.6 \pm 0.9$ \\
\hline & 2.00 & 3.00 & 2.00 & 0.91 & $26,0 \pm 0.7$ & $26.9 \pm 0.7$ \\
\hline & 2.00 & 5.00 & 2.00 & 0.91 & $26.5 \pm 0.5$ & $27.3 \pm 0.5$ \\
\hline \multirow[t]{3}{*}{ EG } & 0.50 & 4.00 & 0.06 & 0.16 & $26.7 \pm 0.8$ & $27.0 \pm 0.8$ \\
\hline & 2.00 & 4.00 & 0.06 & 1.00 & $26.9 \pm 0.5$ & $28.2 \pm 0.5$ \\
\hline & 5.00 & 4.00 & 0.06 & 3.40 & $25.8+0.5$ & $29.0 \pm 0.5$ \\
\hline
\end{tabular}

${ }^{2}$ Calculated from Eq. (9) with $R_{0}=44.5 \AA$.

${ }^{b}$ No correction was included for donor-donor excitation transfer. 


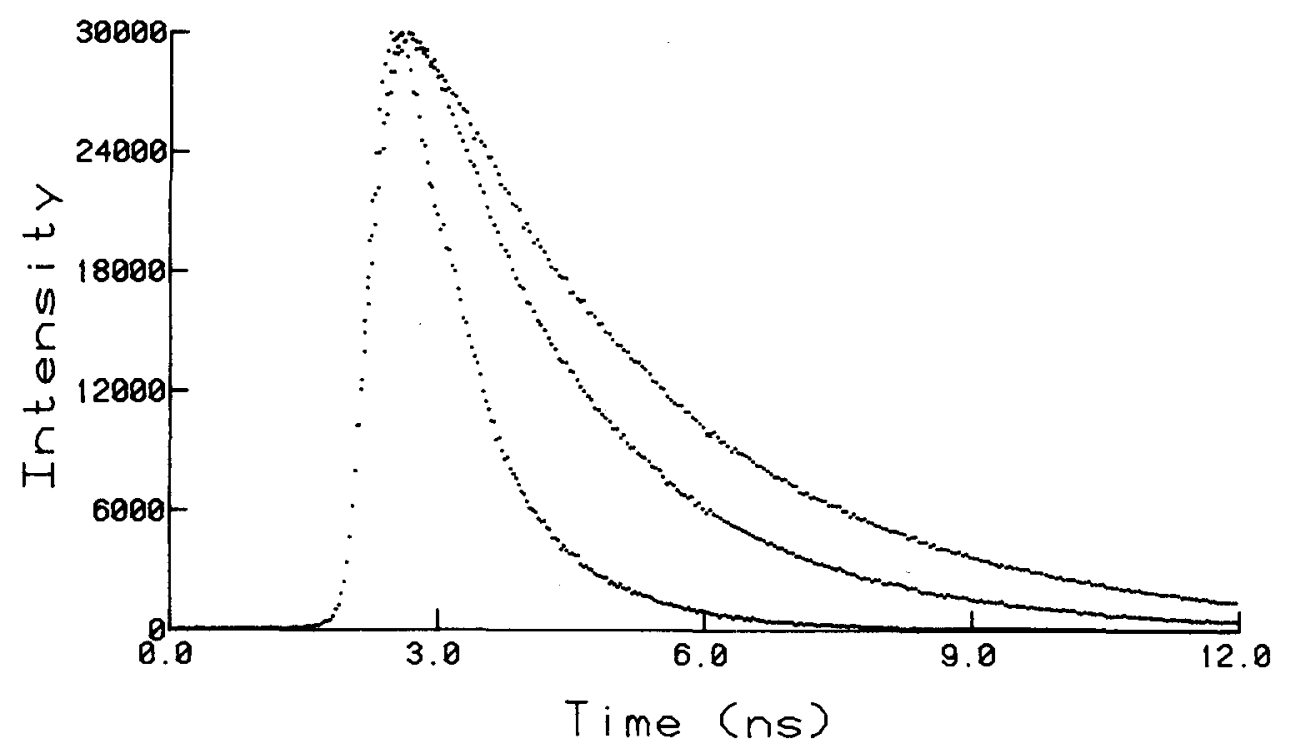

FIG. 5. Fluorescence decays of cresyl violet $\left(2.5 \times 10^{-7} \mathrm{~mol} l^{-1}\right)$ in ethylene glycol containing 5.0 $\times 10^{-5} \mathrm{~mol} \ell^{-1}$ (upper curve), 5.0 $\times 10^{-3} \mathrm{~mol} \mathrm{l}^{-1}$ (middle curve) and $4.0 \times 10^{-2} \mathrm{~mol}^{-1}$ (lower curve) of azulene. The fluorescence was collected through an analyzer set at $54.7^{\circ}$ from the polarization direction of the exciting light.

For methanol however, the no-diffusion equation, Eq. (6) failed to adequately fit the decay curves, especially at the higher acceptor concentrations. Therefore Eq. (8) was used to fit all the fluorescence decay data in both solvents, with the results given in Table II. The quality of the fits was excellent over the entire range of acceptor concentrations in each solvent, as indicated by the reduced chi-squared values $\left(1.0 \leqslant \chi_{r}^{2} \leqslant 1.2\right)$.

The rotational mobility factors presented in Table II indicate that the energy transfer occurs in the dynamic averaging limit at all acceptor concentrations in methanol. In ethylene glycol, the transfer occurs in the intermediate concentration-dependent regime, with $g$ decreasing as the acceptor concentration increases. However, the static averaging limit is never attained in this solvent.

The $R_{0}$ values obtained from the ethylene glycol data are plotted in Fig. 7 against the reduced acceptor centration defined by $C_{\mathrm{A}} / C_{0}\left(C_{\mathrm{A}}\right.$ is the molar acceptor concentration and $C_{0}$ is the critical concentration, $C_{0}$

$\left.=3000 / 2 \pi^{3 / 2} N R_{0}^{3}\right)$. The results are plotted in the form $R_{0} / \bar{R}_{0}$, where $\bar{R}_{0}$ is the average of the $R_{0}$ values obtained at the highest acceptor concentrations. Also plotted in Fig. 7are the results of Porter and Tredwell ${ }^{8}$ for the Rh6G : MG system $\left(\bar{R}_{0}=52.5 \AA\right)$ and of Adams et al. ${ }^{\theta}$ for DODCI : MG $\left(\bar{R}_{0}=45.6 \AA\right)$. Because these results are plotted in reduced units, all three sets may be directly compared. Note that our results span exactly the same range of reduced concentration as Adams et al. ${ }^{3}$; the Rh6G : MG results of Porter and Tredwell ${ }^{8}$ only cover a limited portion of this range. All three sets fall close to 1.0 on the graph at the high end of the range, indicating that $R_{0}$ is independent of $C_{\mathrm{A}}$ in this range. However, the $R_{0}$ values of Adams et al. ${ }^{8}$ increase rapidly as $C_{\mathrm{A}} /$ $C_{0}$ decreases below 0.1 , until $R_{0}$ is some $200 \%$ larger at $C_{\mathrm{A}} / C_{0}=2 \times 10^{-3}$. On the basis of this behavior, these authors concluded that the Förster dipole-dipole rate [Eq. (1)] is not valid at low $C_{\mathrm{A}}$. However, our results for the cresyl violet: azulene system show no deviation: $R_{0}$ is constant to within $10 \%$ over the same range. Referring to the $1 / e$-times in Table II, we note that the ex-

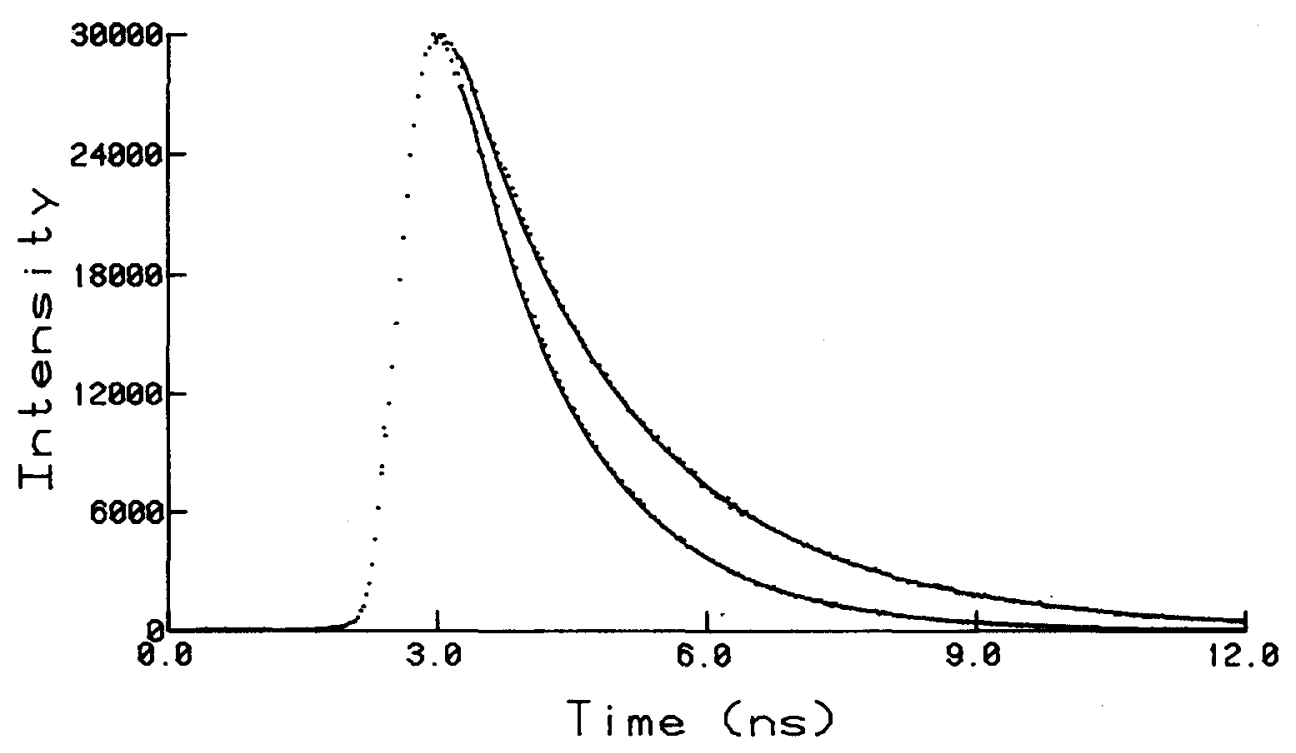

FIG. 6. Fluorescence decays $\left(54.7^{\circ}\right)$ of cresyl violet $\left(2.5 \times 10^{-7}\right.$ mol $\tau^{-1}$ ) containing $1.0 \times 10^{-2}$ mol $f^{-1}$ of azulene in ethylene glycol (upper curve) and in methanol (lower). The points are experimental data and the smooth lines are the best fit of Eq. (8), with $R_{0}=25.7 \AA$ in ethylene glycol and $R_{0}=26.4 \AA$ in methanol. 
TABLE II. Analysis of fluorescence decay curves.

\begin{tabular}{|c|c|c|c|c|c|c|}
\hline \multirow{2}{*}{$\frac{c_{A} / 10^{-3}}{\mathrm{~mol} \sigma^{-1}}$} & \multicolumn{3}{|c|}{ Methanol $^{2}$} & \multicolumn{3}{|c|}{ Ethylene glycol ${ }^{\mathbf{b}}$} \\
\hline & $t_{e} / \mathrm{ps}$ & $g$ & $R_{0} / \AA$ & $t_{e} / \mathrm{ps}$ & $g$ & $R_{0} / \AA$ \\
\hline$\overline{0}$ & 3160 & 0.998 & $\cdots$ & 2870 & 0.973 & $\cdots$ \\
\hline 0.050 & 3119 & 0.998 & $32 \pm 10$ & 2857 & 0.973 & $27 \pm 22$ \\
\hline 0.100 & 3103 & 0.998 & $27.3 \pm 7.0$ & 2842 & 0.973 & $27.7 \pm 11.6$ \\
\hline 0.500 & 2894 & 0.998 & $27.1 \pm 2.5$ & 2756 & 0.972 & $25.9 \pm 2.5$ \\
\hline 1.00 & 2678 & 0.998 & $26.6 \pm 0.6$ & 2610 & 0.972 & $27.4 \pm 1.2$ \\
\hline 2.50 & $\cdots$ & $\cdots$ & ... & 2312 & 0.971 & $26.07 \pm 0.50$ \\
\hline 5.00 & 1569 & 0.998 & $26.36 \pm 0.20$ & 1873 & 0.969 & $25.67 \pm 0.30$ \\
\hline 10.00 & 925 & 0.998 & $26.43 \pm 0.13$ & 1353 & 0.965 & $25.72 \pm 0.19$ \\
\hline 40.00 & 157 & 0.994 & $26.92 \pm 0.10$ & 276 & 0.937 & $25.73 \pm 0.10$ \\
\hline
\end{tabular}

${ }^{2} D_{T}=2.0 \times 10^{-5} \mathrm{~cm}^{2} \mathrm{~s}^{-1}$.

${ }^{b} D_{T}=0.06 \times 10^{-5} \mathrm{~cm}^{2} \mathrm{~s}^{-1}$. Error ranges are $95 \%$ confidence intervals $(\approx 2.8 \sigma)$.

tent of quenching by the acceptors was very small at the lowest acceptor concentrations. Consequently, the decay contains little information on the $\mathrm{D}-\mathrm{A}$ transfer in this regime, and it becomes difficult to determine $R_{0}$ accurately. This is indicated by the magnitude of the error bars for $R_{0}$ shown in Fig. 7. The lifetime and acceptor concentration must be precisely known to accurately determine $R_{0}$ at low $C_{\mathrm{A}} / C_{0}$. The use of a longer lifetime in Eq. (8) can give rise to the same trend evident in data of Adams et al. ${ }^{8}$ For example, if $\tau_{D}=2.98$ ns is used in Eq. (8) (instead of $2.87 \mathrm{ns)} \mathrm{to} \mathrm{fit} \mathrm{our} \mathrm{data}$

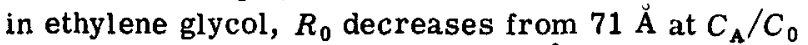
$=2 \times 10^{-3}$ to a limiting value of $26.6 \AA$ when $C_{\mathrm{A}} / C_{0} \mathrm{ex}-$ ceeds 0.1 . Thus an error in $\tau_{D}$ of only $4 \%$ can yield an apparent deviation in our data also, of the same magnitude as reported by Adams et al. ${ }^{9}$ A small increase in the apparent lifetime could be caused by self-absorption effects, as we indeed observed for cresyl violet at concentrations above $10^{-8} \mathrm{~mol} \ell^{-1}$. The donor concentration used by Adams et al. ${ }^{9}$ was $10^{-4} \mathrm{~mol} \ell^{-1}$, suggesting that self absorption was probably the cause of the concentration dependent deviation they observed.

The energy transfer rate in methanol was more rapid than in ethylene glycol as is clearly evident in Fig. 6. The difference between the two decay curves, which have the same acceptor concentration, is mostly due to translational diffusion. Consider the diffusion length given by the Einstein equation $\ell=\left(6 D_{T} \tau_{D}\right)^{1 / 2}$. From the values given in Sec. III, we calculate $\ell=10.2 \AA$ in ethylene glycol and $\ell=61.6 \AA$ in methanol. Thus $\ell \ll R_{0}$ in ethylene glycol and translational diffusion has a minor effect on the donor decay as already noted. However, $\ell \gg R_{0}$ in methanol and considerable diffusion occurs during the energy transfer. The methanol data should thus provide a good opportunity for testing Eq. (8). This equation did indeed fit the methanol decay curves very well over the whole range of $C_{A}$ in Table II. The smooth lines in Fig. 6 are the best fit to Eq. (8) with $R_{0}=26.4 \AA$ in methanol and $R_{0}=25.7 \AA$ in ethylene glycol. Thus the difference between the decay curves in Fig. 6 is accounted for theoretically by Eq. (8) using the calculated translational diffusion coefficients for each solvent. The observation that $R_{0}$ was constant within experimental er ror over the entire range of $C_{A}$ in methanol is further evidence that Eq. (8) accurately accounts for the effects of translational diffusion.

Equation (8) was further verified by the GSR results, summarized in Table I. It is important to recognize a difference between the fluorescence and GSR experiments. In the latter it was necessary to use donor concentrations $C_{\mathrm{D}} \sim 10^{-3} \mathrm{~mol} \mathrm{\ell ^{-1 }}$ in order to detect a reasonable signal. Donor-donor excitation transfer cannot be ignored under these conditions. Assuming for the moment that this transfer is diffusive, its effect can be included by using $D=D_{E}+D_{T}$ when fitting Eq. (8), with the results shown in the second to last column of Table $I$. When the correction for donor-donor transfer was not included, the results shown in the last column were obtained. In methanol $D_{T}>D_{E}$ whenever $C_{D} \leqslant 2 \times 10^{-3}$ mol $\ell^{-1}$ and the donor-donor transfer had a small effect on $R_{0}(\leqslant 1.0 \AA)$. Under these conditions, the GSR decay curves fit Eq. (8) very well; the weighted differences between the theoretical fit and the data, shown in Fig. 3, are almost randomly distributed about zero over the en-

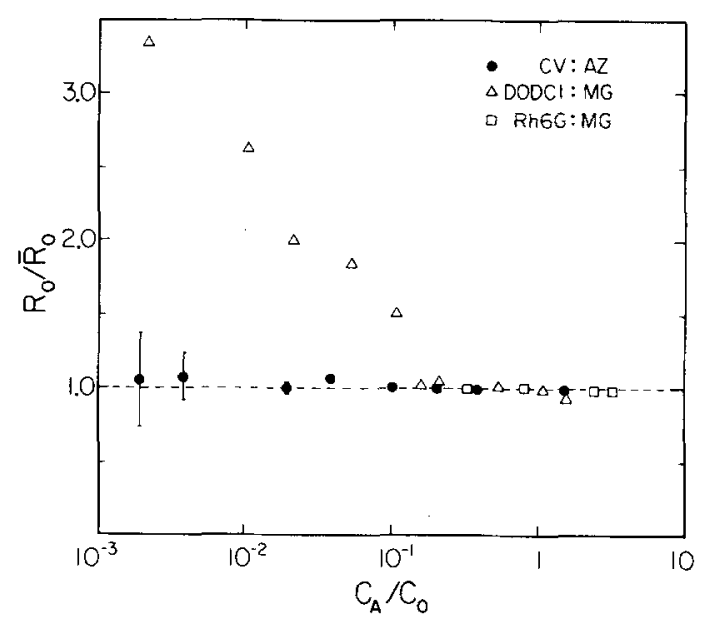

FIG. 7. Critical transfer distance $R_{0}$ as a function of acceptor concentration $C_{\mathrm{A}}$ are shown for three donor: acceptor systems: - Cresyl violet : azulene, this work; $\triangle$ DODCI: malachite green, Adams et al . ${ }^{9}$; व Rhodamine 6G: malachite green, Porter and Tredwell. ${ }^{8}$ The data are plotted in the form $R_{0} \bar{R}_{0}$ versus reduced acceptor concentration $C_{A} / C_{0}$, where $\bar{R}_{0}$ is the critical transfer distance at high acceptor concentrations and $C_{0}$ is the critical concentration (see text for details). 
TABLE III. Summary of critical transfer distances.

\begin{tabular}{lll}
\hline \hline Method & Methanol & Ethylene glycol \\
\hline Fluorescence decay & $26.6 \pm 0.3$ & $25.7 \pm 0.3$ \\
GSR $^{2}$ & $26.2 \pm 0.6$ & $26.4 \pm 0.6$ \\
Spectral'integration & $27.8 \pm 1.0$ & $26.3 \pm 1.0$ \\
\hline
\end{tabular}

${ }^{2}$ Results given here are weighted averages of the results in Tables I and II.

tire time range, in this case $1-160 \mathrm{ps}$.

The donor decay law given by Eq. (8) fits both the GSR and fluorescence decay data for cresyl violet: azulene in methanol, with the same critical transfer distance (Table III). The approximate solution to the combined long-range transfer and diffusion model, derived by Gösele et al.,$^{13-15}$ was found to be accurate in this system over the time range $1 \mathrm{ps}-10 \mathrm{~ns}$. Our results are in accord with the findings of Klein et al. ${ }^{14}$ who also tested Eq. (8) in the case $\ell>R_{0}$, but on a much longer timescale.

Now that we have demonstrated the validity of the diffusion model ${ }^{13-15}$ in treating translational diffusion, we ask whether the model can also be applied to donordonor excitation transfer. This is best studied when translational diffusion is suppressed, such as the GSR results for ethylene glycol, shown in Table I. Obviously, at the highest donor concentration, $C_{D}=5 \times 10^{-3} \mathrm{~mol}^{-1}$, the donor quenching was considerably enhanced, as evident from comparison of the last two columns in Table I. In all cases $D_{E} \gg D_{T}$ in ethylene glycol. The $R_{0}$ values obtained from the GSR curves were almost constant over the range of donor concentrations when both mass and excitation diffusion were included. When donordonor transfer was ignored $R_{0}$ varied with concentration, shown in the last column of Table I. Only by including $D_{E}$ could this dependence on donor concentration be removed, and $R_{0}$ values in reasonable agreement with the fluorescence results be obtained. However, significant deviations were apparent between Eq. (8) and the experimental decay at high donor concentrations; this is clearly evident in the weighted residuals shown in Fig. 4. The GSR signal decays faster than Eq. (8) at short times. The deviation was not evident when donor-donor transfer was effectively absent $\left(C_{\mathrm{D}}<10^{-3} \mathrm{~mol} \ell^{-1}\right)$. Therefore, although Eq. (8), when used with Eq. (10), provides an approximate correction whendonor-donor transfer is present, it does not reproduce the detailed time dependence of the donor decay on the picosecond timescale.

In their theoretical study, Haan and Zwanzig $^{19}$ showed that donor-donor excitation transfer is nondiffusive in dilute solutions at short times. Expanding on this treatment, Gochanour et al. ${ }^{20}$ subsequently calculated the mean-square displacement of the donor excitation for all times and concentrations. Their results demonstrated that the generalized diffusion coefficient, the time derivative of the mean-square displacement, was time dependent for $t \leqslant 2.5 \tau_{D}$ for reduced donor concen- trations $C \leqslant 1.0$. At higher concentrations, $D_{E}$ approached its long time limit more rapidly, and diffusive behavior was established over times of experimental interest. For the data shown in Fig. 4, C=1.1 and nondiffusive behavior is expected. The deviation in Fig. 4 is qualitatively consistent with a time-dependent diffusion coefficient - the donor decay is faster at early times, implying that $D_{E}$ is larger than the long time limit given by Eq. (10). The observed deviations suggest very strongly that donor-donor excitation transfer in this system is nondiffusive over the $160 \mathrm{ps}$ timescale shown in Fig. 4, in accord with the results of Haan and Zwanzig, ${ }^{19}$ and Gochanour et al. ${ }^{20}$

The results from the GSR and fluorescence experiments are in excellent agreement. In Table III we present a summary of our results, giving the average $R_{0}$ value obtained by each transient experiment and from the steady state spectra. Recall that different correction terms have been included in the theoretical fits of the transient data. Even so, the $R_{0}$ values obtained by each method agree very well. This is good evidence that the assumptions underlying the formal equivalence of the GSR and fluorescence experiment, discussed in Sec. II $D$, are valid in this system. Of the three methods listed in Table III, the most accurate is the measurement of fluorescence decays. More importantly, this method allows $R_{0}$ to be obtained over a wide range of acceptor concentrations.

The good agreement between $R_{0}$ from the transient experiments and from the steady-state spectra (Table III) is further evidence that the Förster dipole-dipole model is valid in this system. The excellent fit of the theoretical decay laws to both the fluorescence and GSR curves is also very convincing. We have found that the Förster model was valid over the time range 1 ps -10 ns after excitation, and over a 1000-fold range of acceptor concentrations. This constitutes a considerably more exhaustive test of the Förster model than has previously been performed in time-domain experiments. Remarkably, we could find no deviations from the Förster model, even on the picosecond timescale. Only when donordonor excitation transfer was also present did we detect any deviations.

\section{CONCLUSIONS}

Our main findings may be summarized as follows:

(i) The Förster dipole-dipole model of singlet-singlet donor-acceptor energy transfer accurately reproduces the excited donor decay in cresyl violet: azulene solutions over the time range $1 \mathrm{ps}-10 \mathrm{~ns}$ after excitation.

(ii) The critical transfer distance was constant over a 1000-fold range of acceptor concentrations, proving that the Förster model is valid over this range. We found no evidence of the apparent deviations reported by Adams et al. ${ }^{9}$ at low concentrations for the DODCI : MG system.

(iii) The extension of the Förster model to include the additional time-dependent quenching of the excited donors due to translational diffusion was accurately modeled by the equation of Gösele et al. ${ }^{13-15}$ which is an approxi- 
mate solution to the combined long-range transfer and diffusion equation. This is the first test of this model on the picosecond timescale.

(iv) Donor-donor excitation transfer increases the rate of D-A energy transfer, as does mass diffusion. However, the deviations observed between the GSR data and the diffusion model suggest time-dependent donor excitation diffusion coefficients on the picosecond timescale, in accord with theory. ${ }^{12,13}$

${ }^{1}$ T. Förster, Z. Naturforsch. A 4, 321 (1949); Faraday Discuss. Chem. Soc. 27, 7 (1959).

${ }^{2}$ D. L. Dexter, J. Chem. Phys. 21, 836 (1953).

${ }^{3}$ M. Inokuti and F. Hirayama, J. Chem. Phys. 43, 1978 (1965).

${ }^{4}$ R. G. Bennett, J. Chem. Phys. 41, 3037 (1964).

${ }^{5}$ M. D. Galanin, Sov. Phys. JETP 1, 317 (1955).

${ }^{6}$ A. Blumen and J. Manz, J. Chem. Phys. 71, 4694 (1979).

${ }^{7}$ D. Rehm and K. B. Eisenthal, Chem. Phys. Lett. 9, 387

(1971).

${ }^{8}$ G. Porter and C. J. Tredwell, Chem. Phys. Lett. 56, 278 (1978).

${ }^{9}$ M. C. Adams, D. J. Bradley, W. Sibbett, and J. R. Taylor, Chem. Phys. Lett. 66, 428 (1979).

${ }^{10} \mathrm{P}$. R. Butler and M. J. Pilling, Chem. Phys. 41, 239 (1979).

${ }^{11}$ R. A. Auerbach, G. W. Robinson, and R. Zwanzig, J. Chem. Phys. 72, 3528 (1980).

${ }^{12}$ K. Allinger and A. Blumen, J. Chem. Phys. 72, $4608(1980)$.

${ }^{13}$ U. Gösele, M. Hauser, U. K. A. Klein, and R. Frey, Chem. Phys. Lett. 34, 519 (1975).

${ }^{14}$ U. K. A. Klein, R. Frey, M. Hauser, and U. Gösele, Chem. Phys. Lett. 41, 139 (1976).

${ }^{15}$ U. Gösele, Spectrosc. Lett. 11, 445 (1978).

${ }^{16}$ M. Yokota and O. Tanimoto, J. Phys. Soc. Jpn. 22, 779 (1967).

${ }^{17} \mathrm{~A}$. Blumen, J. Klafter, and R. Silbey, J. Chem. Phys. 72, 5320 (1980).

${ }^{18}$ T. Förster, Ann. Phys. (Leipzig) 2, 55 (1948).

${ }^{18}$ S. W. Haan and R. Zwanzig, J. Chem. Phys. 68, 1879 (1978).

${ }^{20} \mathrm{C}$. R. Gochanour, H. C. Andersen, and M. D. Fayer, J. Chem. Phys, 70, 4254 (1979).

${ }^{21}$ U. Gösele, M. Hauser, and U. K. A. Klein, Z. Phys. Chem. Neue Folge 99, 81 (1978).
${ }^{22}$ M. Leibowitz, J. Phys. Chem. 69, 1061 (1965).

${ }^{23}$ B. Ia. Sveshnikov, Sov. Phys. Dokl. 1, 633 (1956).

${ }^{24}$ I. M. Rozman, Opt. Spectrosc. 4, 536 (1958).

${ }^{25}$ T. R. Waite, Phys, Rev. 107, 463 (1957).

${ }^{26}$ R. E. Dale and J. Eisinger, Biopolymers 13, 1573 (1974).

${ }^{27}$ K. B. Eisenthal and S. Siegel, J. Chem. Phys. 41, 652 (1964); 42, 814 (1965) (E).

${ }^{28}$ I. Z. Steinberg, J. Chem. Phys, 48, 2411 (1968).

${ }^{29} \mathrm{C}$. Bojarski and J. Dudkiewicz, Chem. Phys, Lett. 67, 450 (1979).

${ }^{30} \mathrm{~A}$. Einstein, Investigations on the Theory of Brownian Movement (Dover, New York, 1956).

${ }^{31}$ D. L. Huber, Phys. Rev. B 20, 2307 (1979) and references therein.

${ }^{32}$ T. L. Tao, Biopolymers 8, 609 (1969).

${ }^{33}$ G. R. Fleming, J. M. Morris, and G. W. Robinson, Chem. Phys. 17, 91 (1976).

${ }^{34}$ D. P. Millar, R. Shah, and A. H. Zewail, Chem. Phys. Lett. 66,435 (1979). Equations (8) and (9) of this paper have been reproduced incorrectly. Equation (8) should be

$\left\langle P_{2}\right\rangle=\frac{2}{3}\left\{\frac{1}{4} \exp \left(-6 D_{1} t\right)+\frac{3}{4} \exp \left[-\left(2 D_{\perp}+4 D_{14}\right) t\right]\right\} \quad$;

Eq. (9) should be

$\left.\left\langle P_{2}\right\rangle \approx \exp \left[-3 D_{11}+3 D_{\nu}\right) t\right]$.

${ }^{35} \mathrm{H}$. E. Lessing and A. von Jena, Chem. Phys. Lett. 42, 213 (1976).

${ }^{36}$ C. V. Shank and E. P. Ippen, Appl. Phys. Lett. 24, 373 (1974).

${ }^{37}$ E. P. Ippen and C. V. Shank, Topics in Applied Physics, edited by S. L. Shapiro (Springer, Berlin, 1977), Vol. 18.

${ }^{38}$ K. G. Spears, L. E. Cramer, and L. D. Hoffland, Rev. Sci. Instrum. 49, 255 (1978).

${ }^{39} \mathrm{D}$. Huppert, J. Jortner, and P. Rentzepis, J. Chem. Phys. 56,4826 (1972).

${ }^{40} \mathrm{R}$. J. Robbins, D. P. Millar, and A. H. Zewail, in Topics in Chemical Physics, edited by R. M. Hochstrasser, W. Kaiser, and C. V. Shank (Springer, Berlin, 1980), Vol. 14. ${ }^{41}$ D. P. Millar, R. J. Robbins, and A. H. Zewail, J. Chem. Phys. (submitted for publication).

${ }^{42}$ D. Magde, J. H. Brannon, T. L. Cremers, and J. Olmsted III, J. Phys. Chem. 83, 696 (1979).

${ }^{43}$ A. von Jena and H. E. Lessing, Appl. Phys. 19, 131 (1979).

${ }^{44}$ D. Marquardt, J. SLAM 11, 431 (1963).

${ }^{45}$ T. J. Chuang and K. B. Eisenthal, J. Chem. Phys. 57, 5094 (1972). 